\title{
9. Was tun wir für unsere Gesundheit? Gesundheitsverhalten in der zweiten Lebenshälfte
}

\author{
Svenja M. Spuling, Jochen P. Ziegelmann \& Jenna Wünsche
}

\section{Kernaussagen}

Im Jahr 2014 bestehen deutliche Alters- und Bildungsunterschiede hinsichtlich der sportlichen Aktivität: Ältere sind in geringerem Umfang aktiv als Jüngere. Trotzdem geben noch 29,8 Prozent der 70- bis 85-Jährigen an, mehrmals wöchentlich sportlich aktiv zu sein. Der Anteil mehrmals wöchentlich sportlich aktiver Personen ist bei Hochgebildeten im Vergleich zu Niedriggebildeten doppelt so hoch.

Die sportliche Aktivität hat zwischen 1996 und 2014 kontinuierlich zugenommen: Zwischen 2008 und 2014 konnten jedoch nur in den Altersgruppen ab 60 Jahren weitere Verbesserungen beobachtet werden. Waren beispielsweise im Jahr 2008 noch 13,1 Prozent der 78- bis 83-Jährigen mehrmals wöchentlich sportlich aktiv, so liegt dieser Anteil im Jahr 2014 bereits bei 23,8 Prozent. Demnach haben Altersunterschiede im Anteil sportlich aktiver Personen zwischen 2008 und 2014 abgenommen.

Der Anteil der Raucherinnen und Raucher ist zwischen 2008 und 2014 angestiegen: Dies ist vor allem auf Veränderungen im Rauchverhalten von Frauen zurückzuführen. Der Anteil der Raucherinnen ist zwischen 2008 und 2014 um 3,2 Prozentpunkte angestiegen, während der Anteil der Raucher im gleichen Zeitraum stabil geblieben ist.

Personen, die im Jahr 2014 Vorsorgeleistungen in Anspruch nehmen und Personen, die nicht rauchen, sind auch häufiger regelmäßig sportlich aktiv: Raucherinnen und Raucher sind im Jahr 2014 zu 23,4 Prozent sportlich aktiv, während dieser Anteil bei den Nicht-Raucherinnen und NichtRauchern bei 40,9 Prozent liegt. Personen, die regelmäßig Vorsorgeleistungen in Anspruch nehmen, treiben häufiger mehrmals wöchentlich Sport als Personen, die diese nicht in Anspruch nehmen (Differenz: 9,1 Prozentpunkte). Täglicher Alkoholkonsum hängt dagegen nicht mit regelmäßiger sportlicher Aktivität zusammen.

Personen, die im Jahr 2014 nicht rauchen, sind deutlich häufiger regelmäßig sportlich aktiv als es Nicht-Raucherinnen und Nicht-Raucher im Jahr 2002 waren: Während im Jahr 2002 ein Viertel der Nicht-Raucherinnen beziehungsweise Nicht-Raucher mehrmals wöchentlich sportlich aktiv war, lag dieser Anteil im Jahr 2008 bei knapp einem Drittel und im Jahr 2014 bei 41,2 Prozent. Im Gegensatz dazu beträgt der Anteil sportlich Aktiver unter den Raucherinnen und Rauchern in den Jahren 2002, 2008 und 2014 jeweils etwa 22 Prozent. 


\subsection{Einleitung}

Bis ins hohe Alter hinein ist das eigene Verhalten für die Gesundheit wichtig und stellt damit ein hohes Präventionspotenzial dar (Bundesministerium für Gesundheit 2012). Hinsichtlich der Prävalenz der einzelnen Gesundheits- und Risikoverhaltensweisen bestehen jedoch erhebliche Alters-, Geschlechts- und Bildungsunterschiede, die es in diesem Zusammenhang zu berücksichtigen gilt und deren Herausarbeitung zu einer zielgerichteten Planung von Präventions- und Interventionsmaßnahmen beiträgt. Vor diesem Hintergrund werden im Deutschem Alterssurvey (DEAS) 2014 sowohl Gesundheitsverhaltensweisen wie die sportliche Aktivität und die Inanspruchnahme von Vorsorgeleistungen als auch Risikoverhaltensweisen wie Rauchen und Alkoholkonsum untersucht.

Im Hinblick auf die sportliche Aktivität konnten frühere Untersuchungen zeigen, dass das Alltagsleben schon im jungen Erwachsenenalter häufig von Inaktivität geprägt ist und dass der Anteil sportlich inaktiver Personen mit dem Alter noch weiter zunimmt (Krug, Jordan, Mensink, Müters, Finger, \& Lampert 2013; Wurm, Schöllgen, \& Tesch-Römer 2010; Robert Koch-Institut 2015). Zahllose Studien haben zudem einen Zusammenhang zwischen der sozialen Lage und verschiedenen Gesundheits- und Risikoverhaltensweisen aufzeigen können (zum Beispiel Lampert, von der Lippe, \& Müters 2013b). Die Bildung ist dabei ein wesentliches Merkmal der sozialen Lage und soll in diesem Kapitel als Unterscheidungsmerkmal Berücksichtigung finden. Dabei zeigt sich, dass Personen mit einem höheren Bildungsabschluss häufiger regelmäßig Sport treiben als niedriggebildete Personen (Lampert, Kroll, von der Lippe, Müters, \& Stolzenberg 2013a; Wurm et al. 2010; Robert Koch-Institut 2015). Die Befundlage $\mathrm{zu}$ Geschlechtsunterschieden ist dagegen eher uneinheitlich. Es gibt sowohl Hinweise darauf, dass Frauen sportlich aktiver sind als Männer (Wurm et al. 2010) als auch andersherum, dass Männer eher aktiv sind als Frauen (Krug et al. 2013; Robert Koch-Institut 2015).
Die Inanspruchnahme von Vorsorgeleistungen zählt ebenfalls zu den Gesundheitsverhaltensweisen. Für Ältere ist insbesondere die Grippeschutzimpfung eine wichtige primärpräventive Maßnahme, da für diese Bevölkerungsgruppe ein erhöhtes Risiko besteht, überhaupt beziehungsweise besonders schwer am Influenzavirus zu erkranken. Aus diesem Grund empfiehlt die Ständige Impfkommission (STIKO) die Teilnahme an der jährlichen Grippeschutzimpfung für alle über 60-Jährigen. Es sieht so aus, als würden den Empfehlungen entsprechend insbesondere ältere Erwachsene an einer solchen Impfung regelmäßig teilnehmen, wohingegen Geschlechts- und Bildungsunterschiede eine untergeordnete Rolle spielen (Saß, Wurm, \& Ziese 2009; Robert Koch-Institut 2015). In Deutschland beträgt die jährliche Grippe-Impfquote bei den über 65-jährigen Frauen und Männern ungefähr 60 Prozent und liegt damit deutlich unter der für 2015 von der EU-Kommission angestrebten Impfrate von 75 Prozent für ältere beziehungsweise chronisch kranke Menschen (Robert Koch-Institut 2015).

Im Gegensatz zu primärpräventiven Maßnahmen, die darauf abzielen, dass bestimmte Krankheiten gar nicht erst entstehen, tragen sekundärpräventive Maßnahmen dazu bei, dass bereits bestehende Erkrankungen rechtzeitig erkannt werden. Hierzu zählen Krebsfrüherkennungsuntersuchungen sowie der Gesundheits-Check-Up. Während Frauen, insgesamt betrachtet, häufiger an Krebsfrüherkennungsuntersuchungen teilnehmen, ist eine altersabhängige Zunahme der Inanspruchnahme vor allem ab dem mittleren Erwachsenenalter zu beobachten (Saß et al. 2009; Starker \& Saß 2013). Mit Blick auf den Gesundheits-Check-Up fallen die Teilnahmequoten in höheren Altersgruppen ebenfalls größer aus (Saß et al. 2009). So nahmen laut der GEDA-Studie rund 58 Prozent der Personen ab einem Alter von 65 Jahren an einem Gesundheits-Check-Up teil, während es in der Altersgruppe der 45- bis 54-Jährigen nur rund 49 Prozent waren (Hoebel, Richter, \& Lampert 2013). Für die Krebsfrüherkennungs- 
untersuchungen zeichnet sich darüber hinaus $\mathrm{ab}$, dass Personen mit niedriger Bildung diese seltener in Anspruch nehmen - vermutlich weil sie auch seltener Kenntnis über die Empfehlung der Krankenkassen zur Teilnahme an einer solchen Untersuchung haben (Starker \& Saß 2013). Im Bereich der Vorsorgeuntersuchungen deutet demnach einiges darauf hin, dass diese häufiger von Personen im höheren Alter in Anspruch genommen werden, wobei Bildungs- und Geschlechtsunterschiede nur in der Teilnahme an Krebsfrüherkennungsuntersuchungen $\mathrm{zu}$ erwarten sind.

Präventions- und Interventionsprogramme sind jedoch nicht nur auf die Förderung günstiger Gesundheitsverhaltensweisen, sondern auch auf die Vermeidung von Risikoverhaltensweisen ausgerichtet. Tabak- und Alkoholkonsum stellen hierbei eine besondere Gefahr dar, weil sie noch immer zu den Hauptrisikofaktoren für vorzeitige Mortalität in Europa zählen (WHO 2015). In Bezug auf das Rauchen haben frühere Untersuchungen gezeigt, dass vor allem Personen im jungen und mittleren Erwachsenenalter gefährdet sind, während der Tabakkonsum in älteren Altersgruppen deutlich zurückgeht (Lampert et al. 2013a; Robert Koch-Institut 2015). Verschiedene Studien sind außerdem zu dem übereinstimmenden Ergebnis gekommen, dass Männer sowie Personen mit einem niedrigen Bildungsabschluss häufiger der Gruppe der Raucher angehören als Frauen und Höhergebildete (Lampert et al. 2013b; Wurm et al. 2010; Robert Koch-Institut 2015). Es deutet somit einiges darauf hin, dass Personen mit niedriger Bildung mehrfach gefährdet sind, da sie sowohl seltener sportlich aktiv sind als auch seltener an Krebsfrüherkennungsuntersuchungen teilnehmen und häufiger rauchen (Lampert et al. 2013a; Wurm et al. 2010; Robert Koch-Institut 2015).

Als ein weiteres Risikoverhalten, wurde im DEAS 2014 zum ersten Mal eine Frage zur Häufigkeit des Alkoholkonsums aufgenommen. Es ist bekannt, dass übermäßiger Alkoholkonsum mit Krebs, kardiovaskulären und neuropsychiatrischen Erkrankungen sowie einem erhöhten Mortalitätsrisiko zusammenhängt (Rehm, Mathers, Popova, Thavarncharoensap,
Teerawattananon, \& Patra 2009). Da die WHO mindestens zwei alkoholfreie Tage pro Woche empfiehlt (International Center for Alcohol Policies 2003), wird im vorliegenden Kapitel der tägliche Alkoholkonsum als Risikoverhalten ausgewertet. Bisherige Untersuchungen deuten darauf hin, dass sich der häufig beobachtete $\mathrm{Zu}$ sammenhang zwischen niedriger Bildung und einem ungünstigen Gesundheitsverhalten für den Alkoholkonsum in umgekehrter Weise darstellt. Es scheint, dass eher Personen mit einem hohen sozioökonomischen Status (Bildung) die gesundheitlich verträgliche Alkoholzufuhrmenge besonders häufig überschreiten. Zudem zählen eher die Jüngeren und eher Männer zur Risikogruppe (Burger \& Mensink 2003; Hapke, von der Lippe, \& Gaertner 2013; Robert KochInstitut 2015).

Ein weiterer wichtiger Ansatzpunkt für Interventionen besteht neben der Identifikation von Risikogruppen in der Untersuchung von Zusammenhängen verschiedener gesundheitsbezogener Verhaltensweisen untereinander. Sollten zum Beispiel unterschiedliche Verhaltensweisen miteinander positiv zusammenhängen, könnte dies bedeuten, dass die Verstärkung eines Gesundheitsverhaltens eventuell auch die Verstärkung eines anderen Gesundheitsverhaltens nach sich zieht. Die Befundlage ist jedoch nicht eindeutig: Es ist unklar, ob einzelne gesundheitsbezogene Verhaltensweisen miteinander eher positiv (zum Beispiel Personen, die nicht rauchen, gehören vermehrt in die Gruppe der sportlich Aktiven, da für sie die Aufnahme beziehungsweise Aufrechterhaltung eines sportlich anstrengenden Sportverhaltens mit weniger körperlicher Beanspruchung verbunden ist) oder eher negativ (zum Beispiel sportlich Aktive nehmen weniger Vorsorgeleistungen in Anspruch, da sie sich weniger gesundheitlichen Risiken ausgesetzt fühlen) in Verbindung stehen (Prochaska \& Prochaska 2011). Beide Richtungen sind dabei vorstellbar; ebenso ist es möglich, dass bestimmte Verhaltensweisen unabhängig voneinander sind. Mit den Daten aus dem Jahr 2014 soll deswegen untersucht werden, ob und inwiefern verschiedene gesundheitsbezogene Verhaltensweisen miteinander zusammenhängen. 
Es deutet außerdem einiges darauf hin, dass sich in den letzten Jahren verschiedene Gesundheits- und Risikoverhaltensweisen jeweils zum Positiven gewandelt haben. Besonders erfreulich sind die altersunabhängigen Befunde zum Wandel in der sportlichen Aktivität, die sich bisher in den DEAS-Daten gezeigt haben (Wurm et al. 2010): Immer mehr Menschen in der zweiten Lebenshälfte treiben regelmäßig Sport. Auch bezüglich des Rauchens gibt es Hinweise darauf, dass der Anteil der Raucherinnen und Raucher seit einigen Jahren zurückgeht (Robert Koch-Institut 2015) oder zumindest stabil bleibt (Wurm et al. 2010). Mit der neuen Erhebung aus dem Jahr 2014 soll nun untersucht werden, ob sich dieser günstige Trend fortsetzt und ob er, mit Blick auf die sportliche Aktivität, weiterhin für alle Altersgruppen zu beobachten ist. Außerdem soll der noch unbeantworteten Frage nachgegangen werden, ob sich der Wandel hin zu einem gesundheitsbewussteren Lebensstil auch im Wandel des Zusammenhanges zwischen sportlicher Aktivität und Rauchen

\subsection{Datengrundlage ${ }^{1}$}

Daten. Der Themenbereich Gesundheitsverhalten wurde seit der ersten Erhebung des DEAS im Jahr 1996 sukzessive erweitert und die Abfrage einzelner Gesundheits- und Risikoverhaltensweisen über die Erhebungen hinweg teils angepasst und verändert. Daher können nicht alle Gesundheits- und Risikoverhaltensweisen über alle Erhebungsjahre verglichen werden: Sportliche Aktivität wird in dieser Art und Weise seit 1996 erhoben und Rauchen seit 2002. Die verschiedenen Vorsorgeuntersuchungen sind seit 2008 Bestandteil des DEAS - allerdings wurde die Art der Abfrage zwischen 2008 und 2014 angepasst, weswegen für die Vorsorgeuntersuchungen nur eine Auswertung der aktuellen Erhebung aus dem Jahr 2014 in diesem

1 Die Daten des DEAS können für wissenschaftliche Zwecke kostenlos beim Forschungsdatenzentrum des DZA (www.fdz-dza.de) bezogen werden. widerspiegelt. Das heißt, erhöht sich der Anteil sportlich aktiver Personen in der Gruppe der Raucher beziehungsweise der Nicht-Raucher gleichermaßen oder gibt es hier differenzielle Entwicklungen?

Zusammenfassend werden im vorliegenden Kapitel die folgenden Fragen untersucht:

1. Wie verbreitet sind sportliche Aktivität, die regelmäßige Inanspruchnahme von verschiedenen Vorsorgeleistungen sowie Rauchen und täglicher Alkoholkonsum in der zweiten Lebenshälfte?

2. Wie hängt sportliche Aktivität mit anderen Gesundheits- und Risikoverhaltensweisen zusammen?

3. Wie haben sich sportliche Aktivität und Rauchen über die Zeit gewandelt und zeigt sich dieser Wandel auch im Zusammenhang der beiden Gesundheitsverhaltensweisen?

Kapitel erfolgt. Gleiches gilt für die Abfrage des täglichen Alkoholkonsums, welche erst seit 2014 Bestandteil des DEAS ist. Die sportliche Aktivität wird im Rahmen des persönlichen Interviews erhoben, während die Fragen zur Inanspruchnahme von Vorsorgeuntersuchungen sowie zum Rauchen und zum Alkoholkonsum innerhalb des schriftlichen Fragebogens beantwortet werden.

Sportliche Aktivität. Jede Person wird danach gefragt, ob und wie häufig sie sportlich aktiv ist, „zum Beispiel Wanderungen unternimmt, Fußball spielt, Gymnastik macht oder schwimmen geht". Die Antwortmöglichkeiten sind dabei: ,täglich', ,mehrmals wöchentlich, ,einmal wöchentlich', ,1- bis 3-mal im Monat', ,seltener und ,nie. Für Gruppenunterschiede im Jahr 2014 sowie den Wandel in der sportlichen Aktivität über die Erhebungsjahre werden die Antwortoptionen $\mathrm{zu}$ drei Kategorien zusammen- 
gefasst: ,mehrmals wöchentlich', einmal wöchentlich und ,seltener/nie sportlich aktiv'. Bei Fragestellungen, die sich dem Zusammenhang von sportlicher Aktivität mit anderen Gesundheits- und Risikoverhaltensweisen widmen, wird der Anteil an Personen dargestellt, der mindestens mehrmals wöchentlich sportlich aktiv ist, da empfohlen wird, mehrmals wöchentlich sportlich aktiv zu sein (Jeschke \& Zeilberger 2004). Hierfür wurden die Kategorien ,täglich und ,mehrmals wöchentlich' sportlich aktiv zusammengefasst.

Inanspruchnahme von verschiedenen Vorsorgeleistungen. Das zweite Gesundheitsverhalten, das im vorliegenden Kapitel betrachtet wird, ist die Inanspruchnahme von verschiedenen Vorsorgeleistungen. Im DEAS wird jede Person danach gefragt, ob sie in den letzten Jahren regelmäßig an einer Grippeschutzimpfung, einer Krebsfrüherkennungsuntersuchung oder einem Gesundheits-Check-Up teilgenommen hat. Für Gruppenunterschiede im Jahr 2014 wird für die drei Vorsorgeleistungen jeweils separat der Anteil an Personen berichtet, der daran teilgenommen hat. Zur Beantwortung der Fragestellung nach dem Zusammenhang zwischen der Inanspruchnahme von verschiedenen Vorsorgeleistungen und sportlicher Aktivität wird der Anteil an Personen dargestellt, der mindestens eine der drei untersuchten Vorsorgeleistungen in Anspruch genommen hat.

Rauchen. Die Teilnehmerinnen und Teilnehmer werden darum gebeten, Auskunft über ihren aktuellen Tabakkonsum zu geben. Bei den Ergebnissen zu Gruppenunterschieden im Jahr 2014 sowie bei der Betrachtung des Wandels im Tabakkonsum über die Erhebungsjahre wird der Anteil an Personen, die rauchen, nie geraucht haben und die früher einmal geraucht haben, berichtet. Für die Betrachtung des Zusammenhangs zwischen Rauchen und sportlicher Aktivität, wird der Anteil an Raucherinnen und Rauchern mit dem Anteil an Personen, die nicht rauchen, verglichen, wobei Personen, die nie geraucht haben, sowie ehemalige Raucherinnen und Raucher in einer Gruppe zusammengefasst wurden.
Alkoholkonsum. Als weiteres Risikoverhalten wurde der tägliche Alkoholkonsum ausgewertet. Die Teilnehmerinnen und Teilnehmer werden danach gefragt, wie häufig sie alkoholische Getränke („zum Beispiel Bier, Wein, Sekt, Spirituosen und Mischgetränke") zu sich nehmen. Da die WHO mindestens zwei alkoholfreie Tage pro Woche empfiehlt (International Center for Alcohol Policies 2003), wird im vorliegenden Kapitel der tägliche Alkoholkonsum als Risikoverhalten ausgewertet. Andere alkoholbezogene Risikoverhaltensweisen (wie beispielsweise eine zu hohe Alkoholmenge pro Woche) können mit den Daten des DEAS nicht abgebildet werden.

Gruppierungsvariablen. Um Altersgruppenunterschiede im Jahr 2014 zu veranschaulichen, werden drei Altersgruppen abgebildet (40 bis 54 Jahre, 55 bis 69 Jahre und 70 bis 85 Jahre). Altersunterschiede im Wandel wurden anhand von Sechs-Jahres-Altersgruppen untersucht, um Überschneidungen zwischen Erhebungszeitpunkten und Altersgruppen zu vermeiden (42 bis 47 Jahre, 48 bis 53 Jahre, 54 bis 59 Jahre, 60 bis 65 Jahre, 66 bis 71 Jahre, 72 bis 77 Jahre und 78 bis 83 Jahre). Zur Untersuchung von Bildungsunterschieden wurden - basierend auf einer reduzierten ISCED-Klassifizierung (International Standard Classification of Education, UNESCO 2012) - drei Bildungsgruppen unterschieden: Niedrig-, Mittel- und Hochgebildete (vgl. Kapitel 2).

Analysen. Um Gruppenunterschiede auf Signifikanz zu testen, wurden logistische Regressionen berechnet, wobei für die Stratifizierungsvariablen Altersgruppe, Geschlecht und Region (Ost-/Westdeutschland) kontrolliert wurde. Das genaue Vorgehen ist in Kapitel 2 beschrieben. In den Abbildungen zu Gesundheits- und Risikoverhaltensweisen sowie zum Wandel von sportlicher Aktivität und Tabakkonsum werden gewichtete Häufigkeiten dargestellt. Prozentangaben in den Abbildungen zum Zusammenhang verschiedener Gesundheits- und Risikoverhaltensweisen sowie zum Wandel im Zusammenhang zwischen sportlicher Aktivität und Tabakkonsum stammen dagegen direkt aus den logistischen Regressionen. 


\subsection{Verbreitung verschiedener Gesundheits- und Risikoverhaltensweisen im Jahr 2014}

Im Folgenden wird die Verbreitung der sportlichen Aktivität, des Tabakkonsums sowie der Inanspruchnahme von Vorsorgeleistungen in verschiedenen gesellschaftlichen Gruppen dargestellt.

\section{Im Jahr 2014 bestehen deutliche Alters- und Bildungsunterschiede hinsichtlich der sportlichen Aktivität.}

Im Jahr 2014 sind 35,2 Prozent der 40- bis 85-Jährigen mehrmals wöchentlich sportlich aktiv, weitere 17,4 Prozent treiben immerhin einmal wöchentlich Sport. Allerdings geben 47,4 Prozent an, seltener oder sogar nie sportlich aktiv zu sein (Abbildung 9-1).

Während der Anteil mehrmals wöchentlich sportlich aktiver Personen bei den 55- bis 69-Jährigen etwas größer ausfällt als bei den 40- bis 54-Jährigen, lässt sich in der ältesten Altersgruppe der kleinste Anteil regelmäßig aktiver Personen beobachten. Trotzdem geben noch 29,8 Prozent der 70- bis 85-Jährigen an, mehrmals wöchentlich Sport zu treiben. Dementsprechend gestaltet sich der Trend für die sportliche Inaktivität. Der Anteil an Personen, der seltener als einmal pro Woche oder sogar nie sportlich aktiv ist, liegt bei den 40 - bis 54-Jährigen auf einem etwas höheren Niveau als bei den 55- bis 69-Jährigen. Innerhalb der ältesten Altersgruppe fällt dieser Anteil jedoch am größten aus: Hier sind über die Hälfte der Personen inaktiv. Innerhalb der mittleren Kategorie (einmal wöchentlich sportlich aktiv) finden sich keine signifikanten Altersgruppenunterschiede.
Weiterhin geben 36,4 Prozent der Frauen und 33,9 Prozent der Männer an, mehrmals in der Woche Sport zu treiben. Dagegen ist jeder zweite Mann (51,1 Prozent), aber nur 43,9 Prozent der Frauen sportlich inaktiv. Beide Unterschiede sind statistisch signifikant. Das heißt, Frauen treiben im Durchschnitt häufiger Sport und sind seltener inaktiv als es Männer sind. Es gibt jedoch keine statistisch signifikanten Geschlechtsunterschiede im Anteil der Personen, die sich lediglich einmal wöchentlich sportlich betätigen.

Erwartungsgemäß zeigt sich auch ein deutlicher Bildungseffekt zugunsten höhergebildeter Personen. Während in der niedrigen Bildungsgruppe nur 21,5 Prozent der Personen mehrmals wöchentlich sportlich aktiv sind, steigt dieser Anteil auf 30,6 Prozent in der mittleren Bildungsgruppe an und beträgt in der höchsten Bildungsgruppe 44,7 Prozent. Einmal wöchentlich Sport zu treiben ist in der mittleren und hohen Bildungsgruppe mit 16,8 beziehungsweise 19,4 Prozent ebenfalls verbreiteter als in der niedrigen Bildungsgruppe (12,9 Prozent). Der Unterschied zwischen den beiden oberen Bildungsgruppen ist in dieser Kategorie jedoch nicht signifikant. Dementsprechend nimmt der Anteil an inaktiven Personen mit steigender Bildung ab: Während in der niedrigen Bildungsgruppe fast zwei Drittel der Personen selten oder nie sportlich aktiv sind (65,6 Prozent), trifft dies auf nur noch gut die Hälfte der Personen mit mittlerer Bildung (52,7 Prozent) und etwas mehr als ein Drittel der Personen mit hoher Bildung zu (35,9 Prozent). 
Abbildung 9-1: Sportliche Aktivität, gesamt, nach Alter, Geschlecht und Bildung, im Jahr 2014 (in Prozent)
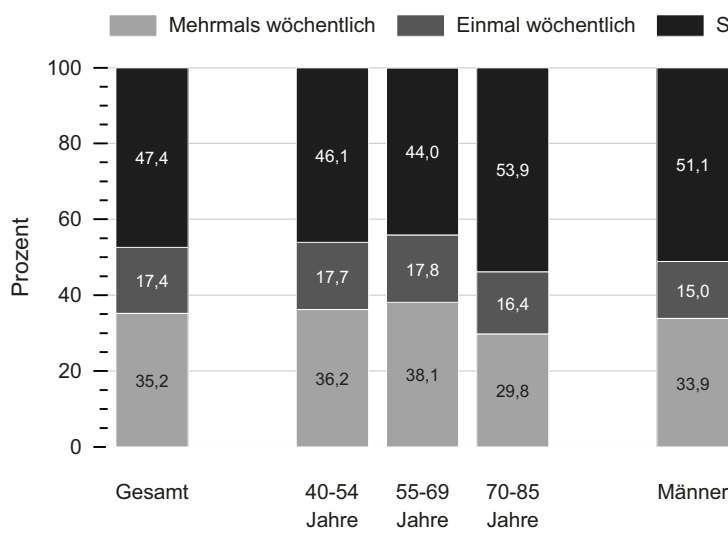

Seltener/Nie

Quelle: DEAS 2014 ( $n=5.998)$, gewichtet, gerundete Angaben; $(p<, 05)$.

Altersgruppen- und Geschlechtsunterschiede sind mit Ausnahme der Kategorie, einmal wöchentlich' signifikant, Bildungsunterschiede sind mit Ausnahme des Unterschiedes zwischen mittlerer und hoher Bildung bei der Kategorie ,einmal wöchentlich` signifikant.

\section{Auch in der Inanspruchnahme von Vorsorgeleistungen bestehen im Jahr 2014 deutliche Unterschiede zwischen den gesellschaftlichen Gruppen.}

33,9 Prozent der 40- bis 85-Jährigen geben 2014 an, in den letzten Jahren regelmäßig eine Grippeschutzimpfung in Anspruch genommen zu haben, 62,9 Prozent haben regelmäßig an einer Krebsfrüherkennungsuntersuchung teilgenommen und 61,9 Prozent haben sich einem Gesundheits-Check-Up unterzogen (Abbildung 9-2).

Abbildung 9-2 veranschaulicht zudem Altersgruppenunterschiede in der Inanspruchnahme der verschiedenen Vorsorgeleistungen. Es wird deutlich, dass in höheren Altersgruppen mehr Personen regelmäßig an einer Grippeschutzimpfung teilnehmen. Während bei den 40 - bis 54-Jährigen 20,4 Prozent regelmäßig eine Grippeschutzimpfung in Anspruch nehmen, ist dieser Anteil bei den 70- bis 85 -Jährigen mehr als doppelt so groß (53,9 Prozent). Insgesamt liegen die geschätzten Impfquoten im DEAS 2014 deutlich unter denen des Robert Koch-Instituts. Dies könnte unter anderem darin liegen, dass im DEAS nach einer regelmäßigen Teilnahme an Grippeschutzimpfungen in den letzten Jahren gefragt wird, während sich das Robert Koch-Institut nur auf die letzte Wintersaison bezieht.

Auch hinsichtlich der Krebsfrüherkennungsuntersuchung und des Gesundheits-CheckUps finden sich Altersunterschiede. Bei der Krebsfrüherkennungsuntersuchung zeigt sich eine stärkere Inanspruchnahme bei den 55-bis 69-Jährigen (69,8 Prozent) im Vergleich zu den 40 - bis 54-Jährigen (57,9 Prozent). In der Gruppe der 70- bis 85-Jährigen liegt die Beteiligung nur bei 62,2 Prozent, befindet sich damit aber immer noch auf einem signifikant höheren Niveau als in der jüngsten Altersgruppe. Die Teilnahmequote beim Gesundheits-Check-Up liegt sowohl in der mittleren als auch in der höchsten Altersgruppe bei ungefähr 66 Prozent. In der jüngsten Altersgruppe geben hingegen nur 55,3 Prozent an, regelmäßig an einem GesundheitsCheck-Up teilgenommen zu haben.

Geschlechtsunterschiede konnten nur für die Krebsvorsorgeuntersuchung und zugunsten der Frauen festgestellt werden: 72,6 Prozent der Frauen und nur 52,6 Prozent der Männer nehmen diese Untersuchungen in Anspruch. Der Anteil an Personen, der an einer Grippeschutzimpfung oder einem Gesundheits-Check-Up teilnimmt, verteilt sich dagegen gleichermaßen über die Geschlechter (vgl. Tabelle 9-2 im Anhang). Bil- 
dungsunterschiede zeigten sich ebenfalls nur bei der Krebsfrüherkennungsuntersuchung: Während Personen mit hoher Bildung häufiger an einer solchen Untersuchung teilnehmen (65,9 Prozent) als Personen mit mittlerer und niedri- ger Bildung (61,5 Prozent beziehungsweise 56,9 Prozent), spielt der Bildungshintergrund für die Teilnahme an einer Grippeschutzimpfung oder einem Gesundheits-Check-Up keine Rolle (ohne Abbildung).

Abbildung 9-2: Anteile der Personen, die regelmäßig an einer Grippeschutzimpfung, einer Krebsvorsorgeuntersuchung und einem Gesundheits-Check-Up teilgenommen haben, gesamt und nach Alter, im Jahr 2014 (in Prozent)

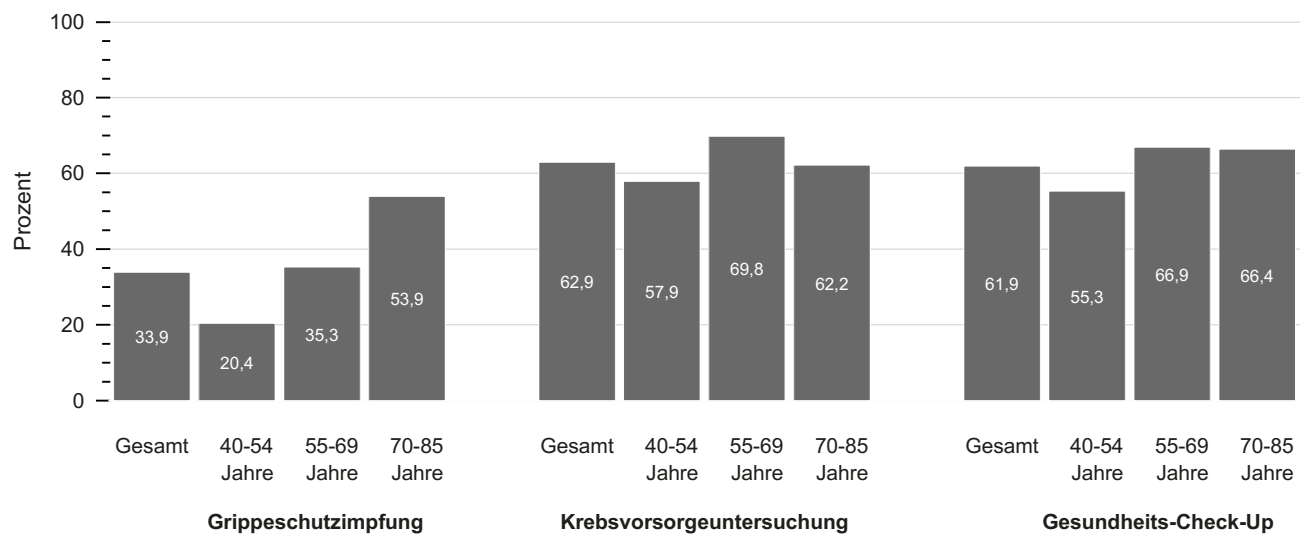

Quelle: DEAS 2014 ( $n=4.181$ für Grippeschutzimpfung; $n=4.144$ für Krebsfrüherkennungsuntersuchung; $n=4.158$ für Gesundheits-Check-Up), gewichtet, gerundete Angaben; $(p<, 05)$.

Unterschiede zwischen den drei Altersgruppen sind mit Ausnahme des Unterschiedes zwischen den 55- bis 69-Jährigen und den 70- bis 85-Jährigen beim Gesundheits-Check-Up signifikant.

\section{Männer sowie Personen im mittleren Erwachsenenalter und Personen mit niedriger bis mittlerer Bildung rauchen am häufigsten.}

Während 23,0 Prozent der 40- bis 85-Jährigen im Jahr 2014 zur Gruppe der Raucherinnen und Raucher gehören, haben 44,1 Prozent nie geraucht, und 32,9 Prozent zählen zu den ehemaligen Raucherinnen und Rauchern (Abbildung 9-3). Abbildung 9-3 gibt weiterhin Auskunft über die Verteilung des Tabakkonsums nach Alter, Geschlecht und Bildung. Erwartungsgemäß zeichnen sich Altersunterschiede für den Tabakkonsum ab: Während von den 40- bis 54-Jährigen etwa jede dritte Person raucht (32,5 Prozent), liegt dieser Anteil bei den 70- bis 85-Jährigen nur bei 8,3 Prozent. Zudem ist der Anteil an Personen, der niemals geraucht hat, in der höchsten Altersgruppe am größten (54,0 Prozent). Frauen rauchen erwartungsgemäß seltener als Männer und gehören häufiger zu denen, die nie geraucht haben beziehungsweise seltener zur Gruppe der ehemaligen Raucherinnen und Raucher. Der erwartete Bildungseffekt zeigt sich bei den aktuellen Raucherinnen und Rauchern nur im Vergleich zur höchsten Bildungsgruppe. Während sich die niedrige Bildungsgruppe $(23,8$ Prozent) und die mittlere Bildungsgruppe (26,5 Prozent) nicht signifikant voneinander unterscheiden, rauchen in der hohen Bildungsgruppe nur 18,3 Prozent. Der Anteil der ehemaligen Raucherinnen und Raucher liegt jedoch bei Personen mit mittlerer und hoher Bildung um fast zehn Prozentpunkte höher als bei den Niedriggebildeten. Niemals geraucht zu haben ist in der niedrigen (51,6 Prozent) und der hohen Bildungsgruppe (48,1 Prozent) am verbreitetsten. In der mittleren Bildungsgruppe (39,9 Prozent) findet sich dagegen der geringste Anteil an NieRauchern. 
Abbildung 9-3: Tabakkonsum, gesamt, nach Alter, Geschlecht und Bildung, im Jahr 2014 (in Prozent)

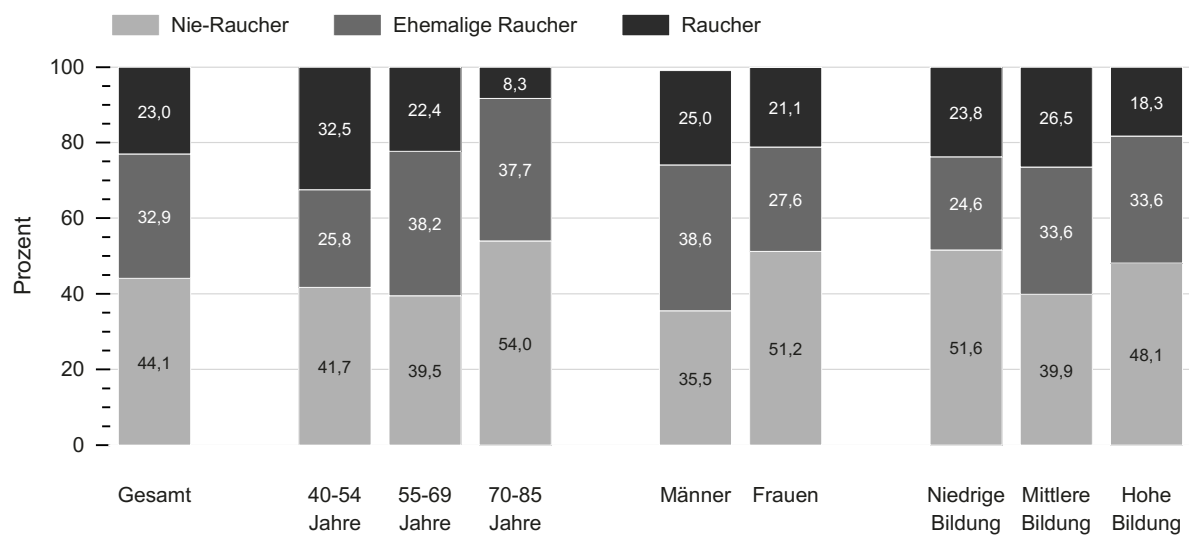

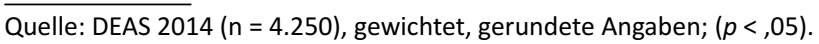

Nie Raucher: Signifikante Altersunterschiede nur zwischen den 70- bis 85 -Jährigen und den anderen Altersgruppen. Signifikante Geschlechtsunterschiede. Signifikante Bildungsunterschiede nur zwischen niedriger und hoher Bildung im Vergleich zu mittlerer Bildung. Ehemalige Raucher: Signifikante Altersunterschiede nur zwischen den 40- bis 54-Jährigen und den beiden anderen Altersgruppen. Signifikante Geschlechtsunterschiede. Signifikante Bildungsunterschiede nur zwischen mittlerer und hoher Bildung im Vergleich zu niedriger Bildung. Raucher: Signifikante Altersunterschiede. Signifikante Geschlechtsunterschiede. Signifikante Bildungsunterschiede nur zwischen niedriger und mittlerer Bildung im Vergleich zu hoher Bildung.

\section{Männer sowie Personen ab 55 Jahren und Personen mit hoher Bildung konsumieren am häufigsten täglich Alkohol.}

Im Jahr 2014 geben 10,9 Prozent der 40- bis 85-Jährigen an, täglich Alkohol zu sich zu nehmen. Dieses Risikoverhalten lässt sich in den höheren Altersgruppen in stärkerem Maße beobachten als in jüngeren Altersgruppen: So liegt der Anteil an Personen, die täglich Alkohol konsumieren in der Gruppe der 40- bis 54-Jährigen bei 7,7 Prozent, während in der Gruppe der 55bis 69-Jährigen und der 70- bis 85 -Jährigen ungefähr 13 Prozent von einem täglichen Alkohol- konsum berichten (Abbildung 9-4). Abbildung 9-4 gibt weiterhin Auskunft über die Verteilung des täglichen Alkoholkonsums nach Geschlecht und Bildung. Dabei wird deutlich, dass vor allem Männer (15,6 Prozent) und nur 6,5 Prozent der Frauen täglich Alkohol zu sich nehmen. Der tägliche Alkoholkonsum unterscheidet sich zwar nicht zwischen der niedrigen und mittleren Bildungsgruppe, aber im Vergleich zur hohen Bildungsgruppe lassen sich statistisch bedeutsame Unterschiede verzeichnen. In der hohen Bildungsgruppe ist der Anteil an Personen, die täglich Alkohol zu sich nehmen, am größten (13,9 Prozent). 
Abbildung 9-4: Anteile der Personen, die täglich Alkohol konsumieren, gesamt, nach Alter, Geschlecht und Bildung, im Jahr 2014 (in Prozent)

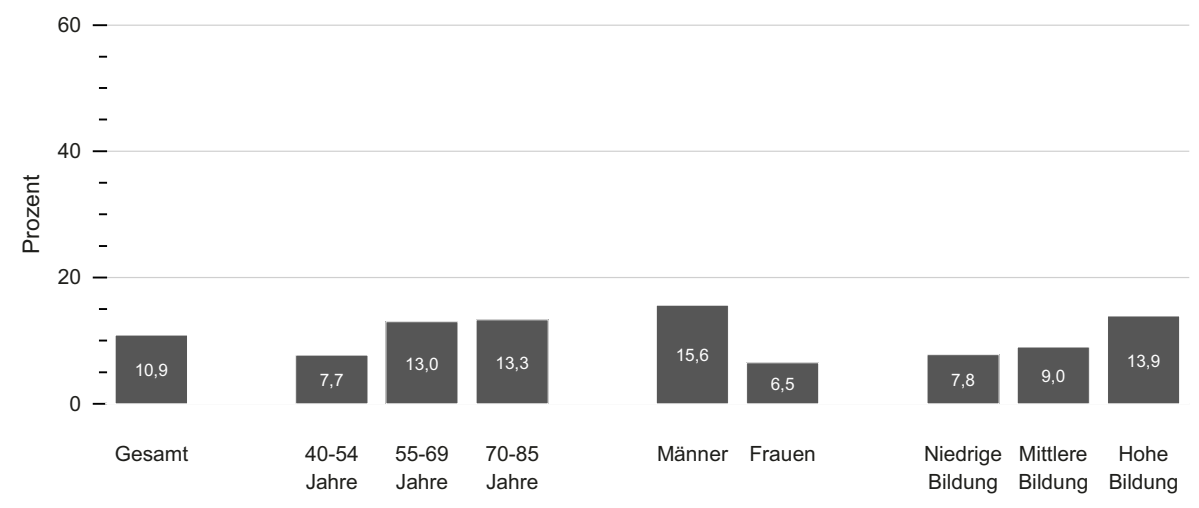

Quelle: DEAS $2014(n=4.221)$, gewichtet, gerundete Angaben; $(p<, 05)$.

Signifikante Altersunterschiede nur zwischen den 40- bis 54-Jährigen und den anderen Altersgruppen. Signifikante Bildungsunterschiede nur zwischen niedriger und mittlerer Bildung im Vergleich zu hoher Bildung.

\subsection{Zusammenhang der sportlichen Aktivität mit anderen Gesundheits- und Risikoverhaltensweisen}

Es wurde der Frage nachgegangen, ob in den Daten von 2014 eine Art übergreifender gesunder Lebensstil beobachtet werden kann, bei dem sportlich aktive Personen beispielsweise vermehrt zur Gruppe der Nicht-Raucherinnen und Nicht-Raucher gehören, regelmäßiger Gesundheitsvorsorgeleistungen in Anspruch nehmen oder seltener täglich Alkohol konsumieren.

\section{Personen, die im Jahr 2014 \\ Vorsorgeleistungen in Anspruch nehmen, und Personen, die nicht rauchen, sind auch häufiger regelmäßig sportlich aktiv.}

Die Ergebnisse, dargestellt in Abbildung 9-5, unterstützen die Annahme eines solchen Le- bensstiles weitestgehend. Der Zusammenhang zwischen sportlicher Aktivität und der regelmäßigen Inanspruchnahme von Vorsorgeleistungen sowie zwischen sportlicher Aktivität und Tabakkonsum ist signifikant. Während lediglich 29,2 Prozent der Personen, die keine Gesundheitsvorsorgeleistung in Anspruch nehmen, mehrmals wöchentlich sportlich aktiv sind, liegt dieser Anteil in der Gruppe der Personen, die regelmäßig mindestens eine Vorsorgeleistung in Anspruch nehmen bei immerhin 38,3 Prozent. Gleichermaßen sind nur 23,4 Prozent der Raucherinnen und Raucher, aber 40,9 Prozent der Nicht-Raucherinnen und Nicht-Raucher mehrmals wöchentlich sportlich aktiv. Dagegen ist der Zusammenhang zwischen regelmäßiger sportlicher Aktivität und täglichem Alkoholkonsum nicht signifikant. 
Abbildung 9-5: Anteile der Personen, die mehrmals wöchentlich sportlich aktiv sind, nach regelmäßiger Inanspruchnahme von Vorsorgeleistungen, Tabakkonsum und täglichem Alkoholkonsum, im Jahr 2014 (in Prozent)

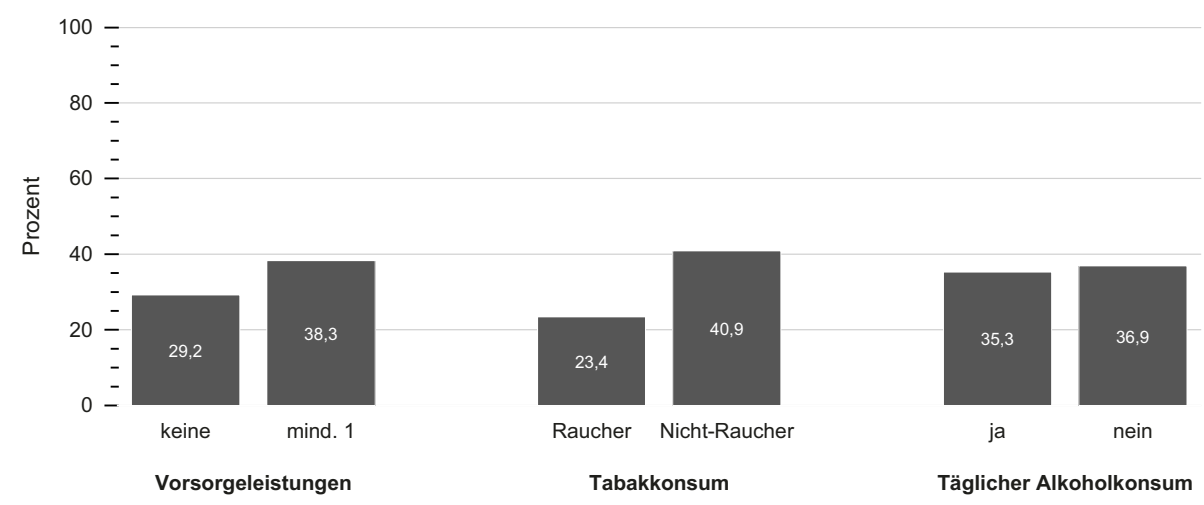

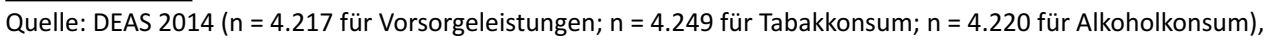
gewichtet, gerundete Angaben; $(p<, 05)$.

Für Vorsorgeleistung und Tabakkonsum signifikante Unterschiede. Für Alkoholkonsum kein signifikanter Unterschied.

\subsection{Wandel in der sportlichen Aktivität und im Tabakkonsum über die Zeit und Wandel des Zusammenhangs dieser beiden Verhaltensweisen}

Es wurde untersucht, ob sich die günstige Entwicklung in der sportlichen Aktivität auch im Jahr 2014 fortgesetzt hat und ob dieser Trend weiterhin für alle Altersgruppen gleichermaßen $\mathrm{zu}$ beobachten ist.

\section{Die sportliche Aktivität hat zwischen 1996 und 2014 kontinuierlich zugenommen.}

Bisher war in den Daten des DEAS von 1996 bis 2008 ein positiver Wandel in der sportlichen Aktivität zu beobachten - und das unabhängig vom Alter (Wurm et al. 2010). Mit der neuen Erhebung im Jahr 2014 zeigt sich zunächst, dass sich der Trend in Richtung einer Zunahme sportlicher Aktivität auch zwischen 2008 und 2014 fortgesetzt hat (vgl. Tabelle A 9-1 im Anhang). Während im Jahr 2008 im Durchschnitt noch 29,4 Prozent der 40- bis 85 -Jährigen berichteten, mehrmals wö- chentlich Sport zu treiben, liegt dieser Anteil im Jahr 2014 bei 35,2 Prozent. Auch die sportliche Inaktivität hat seit 2008 im Durchschnitt weiter abgenommen (von 52,7 Prozent auf 47,4 Prozent). Diese positive Entwicklung zeichnet sich jedoch seit 2008 nicht mehr für alle Altersgruppen in gleicher Weise ab (Abbildung 9-6).

\section{Seit der letzten Befragung im Jahr 2008 konnten weitere Verbesserungen in der sportlichen Aktivität nur in den Altersgruppen ab 60 Jahren beobachtet werden.}

Der Anteil an Personen, der mehrmals wöchentlich sportlich aktiv ist, liegt zwar innerhalb aller Altersgruppen im Jahr 2014 auf einem statistisch bedeutsam höheren Niveau als in der ersten Erhebung im Jahr 1996, es konnten jedoch nur für die Altersgruppen ab 60 Jahren statistisch bedeut- 
same zusätzliche Verbesserungen im sportlichen Aktivitätsverhalten seit 2008 festgestellt werden. In der höchsten Altersgruppe ist der Anteil mehrmals wöchentlich sportlich aktiver Personen seit 2008 sogar um mehr als zehn Prozentpunkte angestiegen (von 13,1 Prozent auf 23,8 Prozent). In den jüngeren Altersgruppen (das heißt 42 bis 47, 48 bis 53 und 54 bis 59 Jahre) ist das Niveau zwischen 2008 und 2014 auf einem stabilen Niveau geblieben, sowohl im Hinblick auf die sportliche Inaktivität als auch in Bezug auf die mehrmals wöchentliche Ausübung sportlicher Aktivität. Zum einen bedeutet dies, dass der positive Wandel hin zu mehr regelmäßiger sportlicher Aktivität und weniger Inaktivität seit 2008 nur noch innerhalb der älteren Altersgruppen ab 60 Jahren $\mathrm{zu}$ beobachten ist und zum anderen weist dieser
Trend darauf hin, dass sich die Altersunterschiede in der sportlichen Aktivität zwischen 2008 und 2014 zugunsten höherer Altersgruppen verkleinert haben.

Zudem haben sich signifikante Geschlechtsunterschiede im Wandel der sportlichen Aktivität gezeigt. Zwar lässt sich sowohl bei Frauen als auch bei Männern ein kontinuierlicher, signifikanter Anstieg in der Ausübung mehrmals wöchentlicher sportlicher Aktivität beziehungsweise eine durchgängige Abnahme in der sportlichen Inaktivität beobachten, bei den Frauen findet diese günstige Entwicklung jedoch in stärkerem Maße statt (ohne Abbildung). Statistisch bedeutsame Bildungsunterschiede ließen sich für den Wandel in der sportlichen Aktivität nicht beobachten.

\section{Abbildung 9-6: Sportliche Aktivität, nach Alter, in den Jahren 1996, 2002, 2008 und 2014 (in Prozent)}

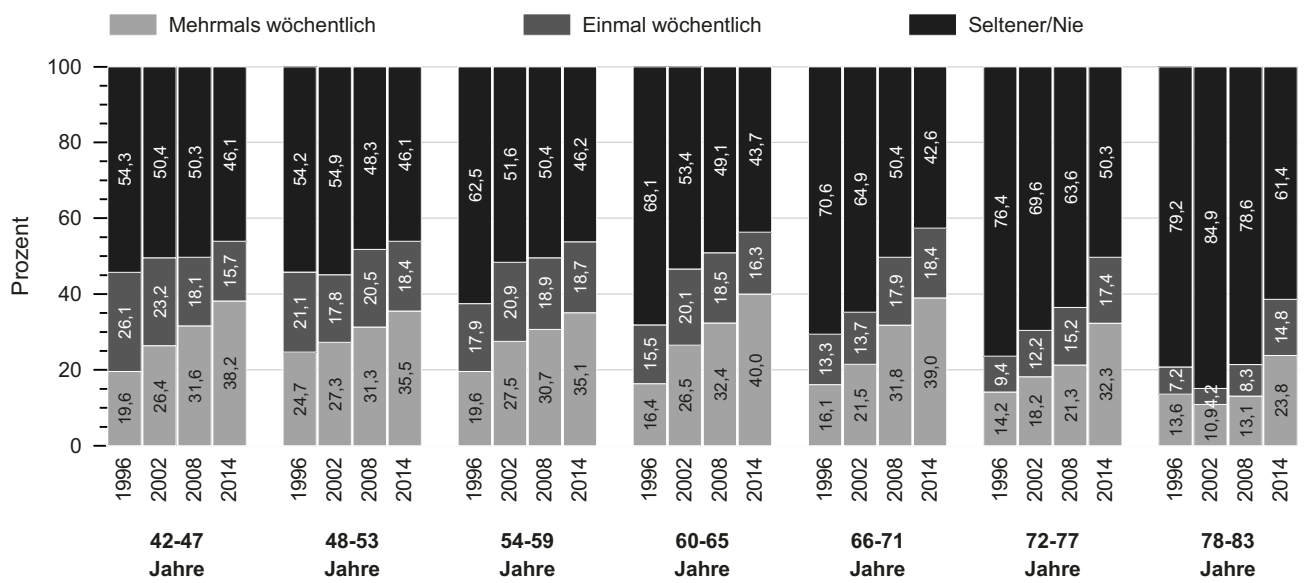

Quelle: DEAS1996 $(n=4.832), 2002(n=3.076), 2008(n=6.202), 2014(n=5.998)$, gewichtet, gerundete Angaben; $(p<, 05)$.

Interaktion zwischen Alter und Erhebungsjahr ist signifikant. Zunahme bei ,mehrmals wöchentlich“ und Abnahme bei ,selten/nie' zwischen 1996 und 2014 in allen Altersgruppen mit Ausnahme der 78- bis 83-Jährigen, dort erst seit 2002. Für ,einmal wöchentlich' zwischen 2002 und 2014 Abnahme bei den Altersgruppen unter 66 Jahren (außer 48 bis 53 Jahre) und Zunahme bei den Altersgruppen ab 66 Jahren. Nur für die Altersgruppen ab 60 Jahren signifikanter Anstieg bei ,mehrmals wöchentlich' bzw. signifikante Abnahme bei ,selten/nie' zwischen 2008 und 2014.

\section{Der Anteil der Raucherinnen und Raucher ist zwischen 2008 und 2014 angestiegen.}

Für den Tabakkonsum ergibt sich eine eher ungünstige Entwicklung seit 2008. Während zwischen 2002 und 2008 der Anteil der 40- bis
85-Jährigen, die rauchen oder früher geraucht haben, insgesamt nicht größer, aber eben auch nicht kleiner geworden ist, hat der Anteil an Raucherinnen und Rauchern über die letzten sechs Jahre, das heißt seit 2008, von 21,2 Prozent auf 23,0 Prozent zugenommen. Im gleichen Zeitraum ist 
auch der Anteil der ehemaligen Raucherinnen und Raucher von 30,1 Prozent auf 32,9 Prozent angestiegen, während das Nie-Rauchen um 4,5 Prozent (von 48,6 Prozent auf 44,1 Prozent) zurückgegangen ist (vgl. Tabelle 9-3 im Anhang).

\section{Dieser ungünstige Wandel im Rauchverhalten ist unter anderem auf Veränderungen im Rauchverhalten von Frauen zurückzuführen.}

Abbildung 9-7 zeigt den Wandel im Rauchen nach Geschlecht, wobei deutlich wird, dass die ungünstige Entwicklung hin $\mathrm{zu}$ mehr Tabakkonsum und weniger Personen, die nie geraucht haben, ausschließlich bei Frauen zu beobachten ist. Im Vergleich zu 2008 ist der Anteil der Raucherinnen um 3,2 Prozent angestiegen (von 17,9 Prozent auf 21,1 Prozent im Jahr 2014), während der Anteil der Frauen, die niemals regelmäßig geraucht haben schon seit 2002 kontinuierlich zurückgegangen ist (von 63,8 Prozent auf 51,2 Prozent im Jahr 2014). Dementsprechend gibt es seit 2002 immer mehr ehemalige Raucherinnen. Dieser Anteil hat sich zwischen 2002 und 2014 um insgesamt 9,1
Prozentpunkte vergrößert. Für die Männer zeigt sich ein anderes Bild. Die Anteile der aktuellen und ehemaligen Raucher sind seit 2008 auf einem stabilen Niveau bei ungefähr 25 beziehungsweise 38 Prozent geblieben. Im Vergleich zu 2002 lässt sich sogar eine günstige Entwicklung beobachten: Der Anteil der ehemaligen Raucher ist von 41,2 Prozent auf 38,6 Prozent im Jahr 2014 zurückgegangen. Diese Ergebnisse deuten darauf hin, dass Frauen sich seit 2002 beziehungsweise 2008 immer mehr dem Tabakkonsum der Männer angleichen, während das Rauchverhalten der Männer eher durch Stabilität oder teilweise sogar durch eine günstige Entwicklung geprägt ist.

Altersgruppenunterschiede konnten für den zeitlichen Wandel im Rauchen nicht beobachtet werden, allerdings zeichnete sich eine differenzielle Entwicklung in Abhängigkeit von der Bildung ab. In der Gruppe der Personen mit niedriger und mittlerer Bildung zeigte sich beispielsweise ein Anstieg im Anteil der Raucherinnen und Raucher zwischen 2002 und 2014, während der Tabakkonsum bei den Hochgebildeten zwischen 2002 und 2008 zurückgegangen und dann stabil geblieben ist (ohne Abbildung).

\section{Abbildung 9-7: Tabakkonsum, nach Geschlecht, in den Jahren 2002, 2008 und 2014 (in Prozent)}

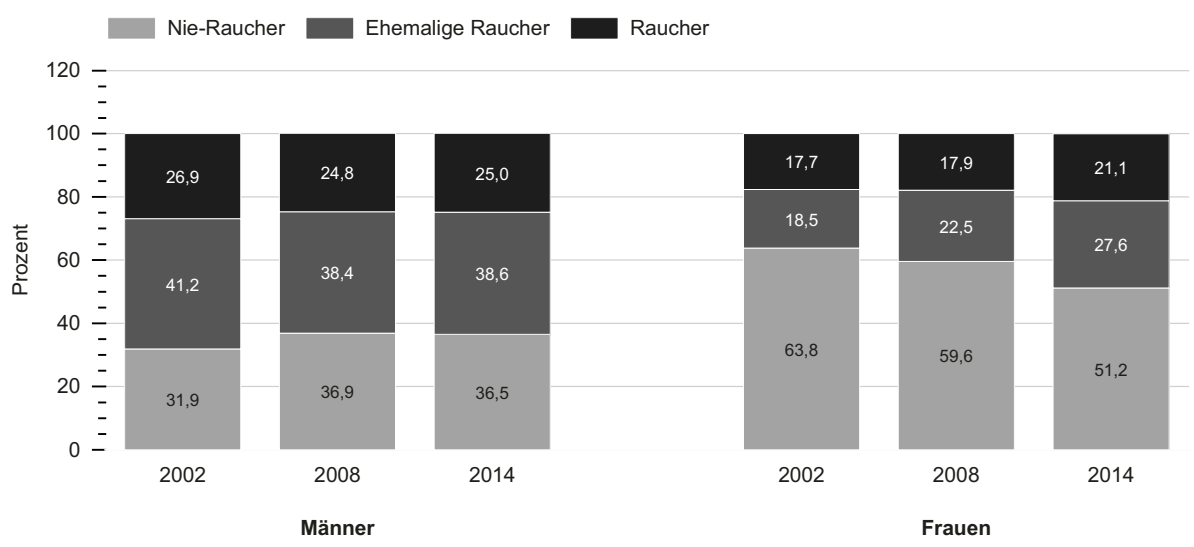

Quelle: DEAS $2002(n=2.743), 2008(n=4.350), 2014(n=4.250)$, gewichtet, gerundete Angaben; $(p<, 05)$. Interaktion zwischen Geschlecht und Erhebungsjahr ist signifikant. Männer: Signifikante Zunahme bei Nie-Rauchern zwischen 2002 und 2008 und Stabilität von 2008 zu 2014, Signifikante Abnahme bei ehemaligen Rauchern zwischen 2002 und 2008 und Stabilität von 2008 zu 2014. Stabilität bei Rauchern. Frauen: Signifikante Abnahme bei den NieRaucherinnen von 2002 zu 2008 und 2008 zu 2014. Signifikante Zunahme der ehemaligen Raucherinnen von 2002 zu 2008 und von 2008 zu 2014. Stabilität der Raucherinnen von 2002 zu 2008 und signifikante Zunahme von 2008 zu 2014. 
Personen, die im Jahr 2014 nicht rauchen, sind deutlich häufiger regelmäßig sportlich aktiv als es Nicht-Raucherinnen und NichtRaucher im Jahr 2002 waren.

Betrachtet man den Zusammenhang zwischen sportlicher Aktivität und Rauchen über die Erhebungen hinweg, zeigt sich, dass nur innerhalb der Gruppe der Nicht-Raucherinnen und Nicht-Raucher der Anteil an Personen, die mehrmals wöchentlich sportlich aktiv sind, zugenommen hat (Abbildung 9-8). Während 2002 nur ein Viertel der Personen, die nicht rauchen, angab, mehrmals wöchentlich sportlich aktiv zu sein (25,1 Prozent), ist dieser Anteil auf 33,3 Prozent im Jahr 2008 und sogar auf 41,2 Prozent im Jahr 2014 angestiegen. Innerhalb der Gruppe der Rauchenden hat sich dagegen der Anteil an Personen, die angeben mehrmals wöchentlich sportlich aktiv zu sein, nicht über den Zeitraum von 2002 bis 2014 verändert.

Abbildung 9-8: Anteile der Personen, die mehrmals wöchentlich sportlich aktiv sind, nach Tabakkonsum, in den Jahren 2002, 2008 und 2014 (in Prozent)

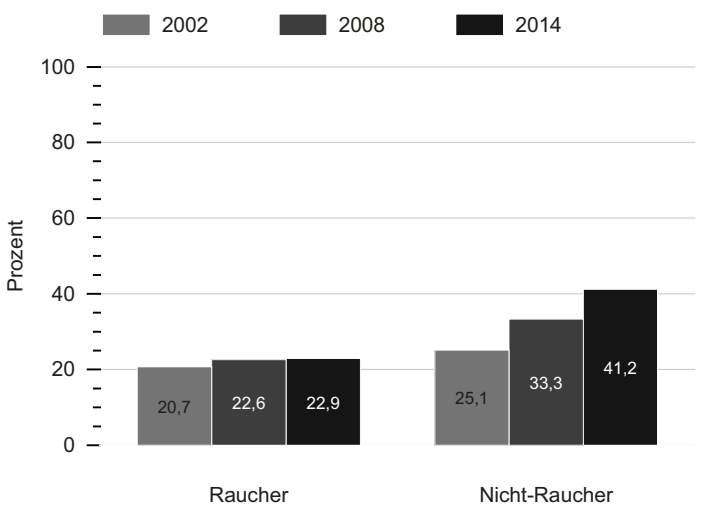

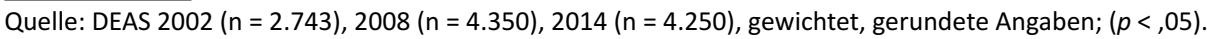
Interaktion zwischen Tabakkonsum und Erhebungsjahr ist signifikant. Raucher: Stabilität über alle Erhebungsjahre. Nicht-Raucher: Signifikante Zunahme von 2002 zu 2008 und 2008 zu 2014.

\subsection{Diskussion und Implikationen}

Bis ins hohe Alter hinein ist das eigene Verhalten für das Auftreten beziehungsweise den Schweregrad von verschiedenen Erkrankungen, für die körperliche Funktionsfähigkeit sowie die subjektive Wahrnehmung der Gesundheit wichtig und stellt damit ein hohes Präventionspotenzial dar. Das heißt, wie wir altern hängt nicht nur von der Gesundheitsversorgung ab, sondern vor allem auch davon, inwieweit wir uns gesundheitsbewusst verhalten und Vorsorgeangebote wahrnehmen und nutzen. Die Politik ist in diesem Zusammenhang angesprochen, nicht nur für Aufklärung über die positiven Auswirkungen von Gesundheitsverhaltensweisen zu sorgen, sondern vor allem auch das Vorhandensein von entsprechenden zielgruppenspezifischen Angeboten weiterhin sicherzustellen beziehungsweise auszuweiten.

Vorliegende Alters-, Bildungs- und Geschlechtsunterschiede entsprechen größtenteils den Erwartungen und lassen darauf schließen, dass vor allem bei Älteren, Niedriggebildeten 
und Männern Präventionspotenziale beziehungsweise Interventionsbedarf bestehen. Weniger als 30 Prozent der Personen innerhalb der ältesten untersuchten Altersgruppe treiben regelmäßig Sport und noch dazu findet sich bei ihnen der größte Anteil an Personen, der täglich Alkohol trinkt.

Personen mit einem niedrigen Bildungsabschluss zählen ebenfalls mit Blick auf die sportliche Aktivität zur Risikogruppe. Hinzu kommt, dass Niedriggebildete im Vergleich zu Personen mit einem hohen Bildungsabschluss seltener Krebsfrüherkennungsuntersuchungen in Anspruch nehmen und zudem den höchsten Anteil an Raucherinnen und Rauchern stellen. Männer zeigen im Vergleich zu Frauen das riskantere Gesundheitsverhalten: Sie sind seltener sportlich aktiv, rauchen häufiger, konsumieren häufiger täglich Alkohol und nehmen seltener an Krebsvorsorgeuntersuchungen teil.

Für die genannten Gruppen ist es daher nicht nur wichtig, dass sie ausreichend über das Vorhandensein bestehender Präventionsangebote informiert werden. Es ist vor allem entscheidend, dass der Zugang zu besagten Maßnahmen möglichst niedrigschwellig hergestellt wird und diese Angebote von den entsprechenden Risikogruppen angenommen und genutzt werden. $\mathrm{Zu}$ beachten ist jedoch, dass sich je nach Kriterium, das innerhalb eines Gesundheits- oder Risikoverhaltens betrachtet wird (zum Beispiel Frequenz versus Dauer der sportlichen Aktivität), unterschiedliche Risikogruppen ergeben können. Beispielsweise gibt es auch Befunde, nach denen Männer eher sportlich aktiv sind als Frauen (Krug et al. 2013; Robert Koch-Institut 2015). Daraus ergäbe sich dann ein besonderer Interventionsbedarf bei den Frauen. Auch der im Rahmen des DEAS 2014 festgestellte Geschlechtsunterschied in der Inanspruchnahme von Krebsfrüherkennungsuntersuchungen sollte vor dem Hintergrund interpretiert werden, dass dieses Verhalten sehr allgemein erfragt wurde. Da es einige geschlechtsspezifische Vorsorgeuntersuchungen gibt (zum Beispiel Brust- und Prostatakrebsvorsorge), lohnt sich auch ein Blick auf die Inanspruchnahme von Früherkennungsmaßnahmen, welche für beide Geschlechter gleichermaßen empfohlen werden. So nahmen beispielsweise laut Daten des Robert Koch-Instituts aus dem Jahr 2010 Frauen und Männer ungefähr gleichhäufig (32,9 Prozent der Frauen und 33,9 Prozent der Männer) an Hautkrebsfrüherkennungsuntersuchungen sowie an einer Darmspiegelung (rund 58 Prozent der über 55-Jährigen Männer und Frauen) teil. Es könnte vermutet werden, dass sich Geschlechtsunterschiede in diesem Vorsorgeverhalten vor allem auf geschlechtsspezifische Krebsfrüherkennungsuntersuchungen beziehen und dass beispielsweise gezielte Kampagnen zur Förderung der Prostatakrebsvorsorge besonders sinnvoll sein könnten. Innerhalb solcher Kampagnen ist es jedoch wichtig, in anschaulicher und nachvollziehbarer Weise über die KostenNutzen-Beziehung entsprechender Vorsorgemaßnahmen aufzuklären, da nicht alle Kontrolluntersuchungen in gleichem Maße von Vorteil sind. Im Hinblick auf die Ultraschall-Diagnostik zur Früherkennung von Eierstockkrebs konnte beispielsweise gezeigt werden, dass diese Untersuchungen eher schädlich als nützlich sind: Die Sterberate an Eierstockkrebs wird durch den Ultraschall nicht verringert, stattdessen kommt es häufig zu Fehlalarmen, die mit unnötigen medizinischen Eingriffen und weiteren Nebenwirkungen einhergehen (Gigerenzer 2015).

Für den Wandel im Gesundheitsverhalten findet sich im DEAS 2014 erfreulicherweise eine Fortsetzung der Zunahme sportlicher Aktivität. Es ist jedoch hervorzuheben, dass dieser Trend seit 2008 in erster Linie die Altersgruppen ab 60 Jahren betrifft. Vorstellbar wäre, dass diese differenzielle Entwicklung dadurch zustande gekommen ist, dass ältere Personen in den letzten Jahren mehr und mehr in den Fokus der Gesundheitsförderung gerückt sind, oder dass der verbesserte gesundheitliche Zustand dieser Gruppe die vermehrte Ausübung von sportlicher Aktivität begünstigt. Trotzdem sind hier auch weiterhin große Präventionspotenziale festzustellen, da immer noch deutlich mehr als die Hälfte der Bevölkerung nicht ausreichend aktiv ist.

Mit Blick auf die Entwicklung des Tabakkonsums berichteten das Robert Koch-Institut und der Mikrozensus einheitlich von einer leicht sinkenden Raucherquote seit 2003 (Fleitmann, 
Dohnke, Balke, Rustler, \& Sonntag 2010; Robert Koch-Institut 2015). Dieser günstige Trend kann anhand der DEAS-Daten aus dem Jahr 2014 nicht unterstützt werden: Während sich zwischen 2002 und 2008 keine Veränderungen gezeigt haben, hat der Tabakkonsum seit 2008 zugenommen. Dieser Befund lässt sich unter anderem auf ein verändertes Rauchverhalten bei Frauen zurückführen. Im Vergleich zu den beiden früheren Erhebungen geben Frauen im Jahr 2014 beispielsweise häufiger an zu rauchen, während der Raucheranteil bei den Männern seit 2002 stabil bei etwa einem Viertel liegt. In anderen Worten: Frauen nähern sich dem Tabakkonsum der Männer an. Im Hinblick darauf, dass sie besonders sensibel auf die schädlichen Wirkungen des Tabakrauchs reagieren und sich bereits ein Anstieg in der Lungenkrebsprävalenz bei Frauen abzeichnet (Neubauer, Welte, Beiche, Koenig, Buesch, \& Leidl 2006), ist dieser Trend besonders alarmierend. Zukünftige Maßnahmen zur Senkung des Tabakkonsums sollten daher ein besonderes Augenmerk auf rauchende Frauen legen.

Besorgniserregend ist zudem, dass sich bei Raucherinnen und Rauchern kein positiver Wandel in der sportlichen Aktivität zeigt. Personen, die nicht rauchen, werden dagegen immer aktiver. Diese Befunde deuten darauf hin, dass bei Nicht-Raucherinnen und Nicht-Rauchern das Gesundheitsbewusstsein über die Zeit hinweg steigt, während sich bei Raucherinnen und Rauchern diesbezüglich kein Wandel abzeichnet.

Zudem zeigt sich ein positiver Zusammenhang zwischen sportlicher Aktivität und der Inanspruchnahme von Vorsorgeleistungen sowie ein negativer Zusammenhang zwischen sportlicher Aktivität und Rauchen. Damit liefern die Befunde einen weiteren Hinweis auf den synergetischen Zusammenhang verschiedener Gesundheitsverhaltensweisen, sie lassen jedoch aufgrund ihrer querschnittlichen Natur keine Aussagen über kausale Wirkbeziehungen zu. Erklären kann man solche synergistischen $\mathrm{Zu}$ sammenhänge, wie zum Beispiel den zwischen sportlicher Aktivität und Rauchen (Nagaya, Yoshida, Takahashi, \& Kawai 2007), damit, dass sportliche Aktivität bei Rauchern das Verlangen nach Nikotin verringert (Taylor, Ussher, \&
Faulkner 2007). Auf solchen synergistischen Zusammenhängen baut die ,Gateway-Hypothe$\mathrm{se}^{`}$ auf, nach der Veränderungen des Verhaltens (zum Beispiel sportliche Aktivität) auch Veränderungen bei anderen Verhaltensweisen (zum Beispiel Ernährung) nach sich ziehen. Statt bei mehreren Verhaltensweisen $\mathrm{zu}$ intervenieren, konzentriert man sich auf ein Verhalten und erhofft dadurch die Chance zu erhöhen, dass auch ein damit zusammenhängendes Verhalten positiv verändert wird. Entsprechende Interventionsstudien sind jedoch noch sehr selten und liefern bisher keine eindeutigen Befunde (siehe zum BeispielDutton, Napolitano, Whiteley, \& Marcus 2008). Möglicherweise lassen sich weitere Nachweise für die ,Gateway-Hypothese erbringen, wenn man nicht nur Synergien zwischen den klassischen beziehungsweise proximalen Gesundheitsverhaltensweisen (zum Beispiel sportliche Aktivität) in Betracht zieht, sondern auch distalere Gesundheitsverhaltensweisen, wie zum Beispiel soziale Partizipation (Ziegelmann \& Knoll 2015), in solche Analysen miteinbezieht. In diesem Sinne könnte die soziale Partizipation in Form von freiwilligem Engagement in Interventionen angegangen werden, um damit als positiven Nebeneffekt auch die sportliche Aktivität zu steigern (z.B. Baltimore Experience Corps Trial: Tan, Rebok, Yu, Frangakis, Carlson, Wang, Ricks, Tanner, Mc-Gill, \& Fried 2009). Gerade für ältere Erwachsene werden derartige Interventionsansätze mit Erfolg genutzt, da reine Gesundheitsverhaltensinterventionen nicht von allen älteren Menschen als attraktiv angesehen werden.

Zwischen sportlicher Aktivität und Alkoholkonsum konnte kein Zusammenhang nachgewiesen werden. Immerhin ist aber festzuhalten, dass kein positiver Zusammenhang besteht, wie er von anderen Autoren in einem systematischen Review für alle Altersgruppen dokumentiert wurde (Piazza-Gardner \& Barry 2012). Interessant ist außerdem, dass innerhalb des DEAS 2014 die Personengruppe mit dem höchsten Bildungsstand zwar die niedrigste Raucher-, jedoch die höchste Alkoholquote aufweist, was wiederum einen Hinweis dafür liefert, dass Risikoverhaltensweisen nicht un- 
bedingt in positiver Weise miteinander zusammenhängen müssen.

Für die erfolgreiche Umsetzung des Nationalen Gesundheitsziels ,Gesund älter werden' (Bundesministerium für Gesundheit 2012) sollten somit nicht nur Risikogruppen, sondern auch sy- nergistische Zusammenhänge zwischen verschiedenen Gesundheitsverhaltensweisen im Blick behalten werden, da sie einen weiteren wichtigen Ansatzpunkt für die Gestaltung von Präventionsund Interventionsprogrammen liefern.

\section{Literatur}

Bundesministerium für Gesundheit (2012). Nationales Gesundheitsziel: Gesund älter werden. Berlin: Bundesministerium für Gesundheit.

Burger, M., \& Mensink, G. (2003). Bundes-Gesundheitssurvey: Alkohol. Berlin: Robert Koch-Institut.

Dutton, G. R., Napolitano, M. A., Whiteley, J. A., \& Marcus, B. H. (2008). Is physical activity a gateway behavior for diet? Findings from a physical activity trial. Preventive Medicine, 46, 216-221.

Fleitmann, S., Dohnke, B., Balke, K., Rustler, C., \& Sonntag, U. (2010). Frauen und Rauchen. Bundesgesundheitsblatt - Gesundheitsforschung - Gesundheitsschutz, 53(2), 117-124. doi: 10.1007/s00103-009-1005-3.

Gigerenzer, G. (2015). Statement zu "Faktenboxen". Pressekonferenz "Faktenboxen" des AOK-Bundesverbandes. Berlin: AOK.

Hapke, U., Lippe, E. von der, \& Gaertner, B. (2013). Riskanter Alkoholkonsum und Rauschtrinken unter Berücksichtigung von Verletzungen und der Inanspruchnahme alkoholspezifischer medizinischer Beratung. Bundesgesundheitsblatt, 56, 809-813. doi: 10.1007/ s00103-013-1699-0.

Hoebel, J., Richter, M., \& Lampert, T. (2013). Social status and participation in health checks in men and women in Germany: Results from the German Health Update (GEDA), 2009 and 2010. Deutsches Ärzteblatt International, 110(41), 679-685. doi: 10.3238/ arztebl.2013.0679.

International Center for Alcohol Policies (2003). International Drinking Guidelines (ICAP Report No. 14). Washington DC: ICAP.

Jeschke, D., \& Zeilberger, K. (2004). Altern und körperliche Aktivität. Deutsches Ärzteblatt, 101(12), A 789 - A 798.

Krug, S., Jordan, S., Mensink, G. B. M., Müters, S., Finger, J. D., \& Lampert, T. (2013). Körperliche Aktivität. Bundesgesundheitsblatt, 56, 765-771. doi: 10.1007/ s00103-012-1661-6.

Lampert, T., Kroll, L. E., Lippe, E. von der, Müters, S., \& Stolzenberg, H. (2013a). Sozioökonomischer Status

und Gesundheit. Bundesgesundheitsblatt, 56, 814821. doi: 10.1007/s00103-013-1695-4.

Lampert, T., Lippe, E. von der, \& Müters, S. (2013b). Verbreitung des Rauchens in der Erwachsenenbevölkerung in Deutschland. Bundesgesundheitsblatt, 56, 802-808. doi: 10.1007/s00103-013-1698-1.

Nagaya, T., Yoshida, H., Takahashi, H., \& Kawai, M. (2007). Cigarette smoking weakens exercise habits in healthy men. Nicotine \& Tobacco Research, 9, 1027-1032.

Neubauer, S., Welte, R., Beiche, A., Koenig, H. H., Buesch, K., \& Leidl, R. (2006). Mortality, morbidity and costs attributable to smoking in Germany: update and a 10year comparison. Tobacco Control, 15(6), 464-471. doi: 10.1136/tc.2006.016030.

Piazza-Gardner, A. K., \& Barry, A. E. (2012). Examining physical activity levels and alcohol consumption: are people who drink more active? American Journal of Health Promotion, 26, e95-104. doi: 10.4278/ ajhp.100929-LIT-328.

Prochaska, J. J., \& Prochaska, J. O. (2011). A review of multiple health behavior change interventions for primary prevention. American Journal of Lifestyle Medicine, 5.

Rehm, J., Mathers, C., Popova, S., Thavarncharoensap, M., Teerawattananon, Y., \& Patra, J. (2009). Global burden of disease and injury and economic cost attributable to alcohol use and alcohol-use disorders. Lancet, 373(9682), 2223-2233.

Robert Koch-Institut (2015). Gesundheit in Deutschland 2015. Berlin: Robert Koch-Institut.

Saß, A.-C., Wurm, S., \& Ziese, T. (2009). Inanspruchnahmeverhalten. In: K. Böhm, C. Tesch-Römer \& T. Ziese (Hrsg.) Gesundheit und Krankheit im Alter (S. 134-159). Berlin: Robert Koch-Institut.

Starker, A., \& Saß, A.-C. (2013). Inanspruchnahme von Krebsfrüherkennungsuntersuchungen. Bundesgesundheitsblatt, 56, 858-867. doi: 10.1007/s00103-0121655-4.

Tan, E. J., Rebok, G. W., Yu, Q., Frangakis, C. E., Carlson, M. C., Wang, T., Ricks, M., Tanner, E. K., Mc-Gill, S., \& 
Fried, L. P. (2009). The long-term relationship between high-intensity volunteering and physical activity in older African American women. Oxford: University Press.

Taylor, A. H., Ussher, M. H., \& Faulkner, G. (2007). The acute effects of exercise on cigarette cravings, withdrawal symptoms, affect and smoking behaviour: a systematic review. Addiction, 102, 534-543.

UNESCO (2012). International Standard Classification of Education ISCED 2011. Montreal: UNESCO Institute for Statistics.
WHO (2015). Europäischer Gesundheitsbericht 2015. Kopenhagen: Weltgesundheitsorgansitation Regionalbüro für Europa.

Wurm, S., Schöllgen, I., \& Tesch-Römer, C. (2010). Gesundheit. In: A. Motel-Klingebiel, S. Wurm \& C. Tesch-Römer (Hrsg.) Altern im Wandel. Befunde des Deutschen Alterssurveys (DEAS) (S. 90-117). Stuttgart: Kohlhammer.

Ziegelmann, J. P., \& Knoll, N. (2015). Future directions in the study of health behavior among older adults. Gerontology, 61, 469-476. doi: 10.1159/000369857.

Open Access Dieses Kapitel wird unter der Creative Commons Namensnennung 2.5 International Lizenz (http://creativecommons.org/licenses/by/2.5/deed.de) veröffentlicht, welche die Nutzung, Vervielfältigung, Bearbeitung, Verbreitung und Wiedergabe in jeglichem Medium und Format erlaubt, sofern Sie den/die ursprünglichen Autor(en) und die Quelle ordnungsgemäß nennen, einen Link zur Creative Commons Lizenz beifügen und angeben, ob Änderungen vorgenommen wurden.

Die in diesem Kapitel enthaltenen Bilder und sonstiges Drittmaterial unterliegen ebenfalls der genannten Creative Commons Lizenz, sofern sich aus der Abbildungslegende nichts anderes ergibt. Sofern das betreffende Material nicht unter der genannten Creative Commons Lizenz steht und die betreffende Handlung nicht nach gesetzlichen Vorschriften erlaubt ist, ist für die oben aufgeführten Weiterverwendungen des Materials die Einwilligung des jeweiligen Rechteinhabers einzuholen. 